\title{
Practical methods for refeeding patients with anorexia
}

\section{nervosa}

\author{
Koutsavlis $\mathrm{A}^{1}{ }^{*}$, Forzley EM ${ }^{1}$, Johnson $\mathrm{M}^{1}$, Oakley MA ${ }^{1}$, Trees $\mathrm{N}^{1}$ and Mehler $\mathrm{P}^{1-3}$ \\ ${ }^{1}$ Eating Recovery Center, 1830 Franklin Street Denver, CO 80218, USA \\ ${ }^{2}$ Department of Medicine, University of Colorado Health Sciences Center, USA \\ ${ }^{3}$ ACUTE at Denver Health 777 Bannock Street, MC4000, Denver, CO 80204, USA
}

\begin{abstract}
Anorexia nervosa (AN) is a complex mental health disorder that may result in life threatening medical complications if not properly treated. Refeeding and weight restoration are the primary goals of treatment in AN. A multidisciplinary team is essential during this process in order to properly monitor and address complications that can arise during refeeding. There are benefits and drawbacks to different approaches to refeeding and weight restoration, however, recent research supports more aggressive approaches to refeeding and weight restoration. This review explores recommendations for methods of refeeding from various professional organizations, current standards of practice, as well as management of complications that may arise during the refeeding process.
\end{abstract}

\section{Abbreviations and symbols}

AN: Anorexia nervosa; AN-R: Anorexia nervosa restricting type; AN-BP: Anorexia nervosa binge-eating/purging type; BMI: Body mass index; RD: Registered dietitian; AND: Academy of nutrition and dietetics; NICE: National institute for health and clinical excellence; ATP: Adenosine-5-triphosphate; 2-3 DPG: 2,3-diphosphoglycerate; SMA: Superior mesenteric artery; GI: gastrointestinal; CT: Computerized tomography

\section{Introduction}

The Diagnostic and Statistical Manual of Mental Disorders, $5^{\text {th }}$ Edition defines anorexia nervosa (AN) as the restriction of energy intake relative to requirements leading to significantly low body weight, in conjunction with an extreme fear of gaining weight and a distorted view of body weight and shape [1]. AN is defined as either restricting type (AN-R) or binge-eating/purging type (AN-BP). AN-R occurs "when weight loss is accomplished primarily through dieting, fasting and/or excessive exercise". AN-BP is diagnosed "when during the last three months the individual has engaged in recurrent episodes of binge eating or purging behavior (i.e., self-induced vomiting or misuse of laxatives, diuretics, or enemas)" The severity of AN is defined by body mass index (BMI): mild: BMI > $17 \mathrm{~kg} / \mathrm{m}^{2}$, moderate: BMI 16-16.99 kg/ $\mathrm{m}^{2}$, severe: BMI $15-15.99 \mathrm{~kg} / \mathrm{m}^{2}$, and extreme: $\mathrm{BMI}<15 \mathrm{~kg} / \mathrm{m}^{2}$.

The overall prevalence of $\mathrm{AN}$ is estimated to be $1-2 \%$ of females between the ages of $15-35$. Males have a rate of $0.1 \%$ and the overall female to male ratio is 1:20 [2-4]. AN has the highest mortality rate of any psychiatric disorder, with a reported mortality rate of $5.6 \%$ [5]. Medical complications related to the disorder result in $50 \%$ of the deaths in individuals with $\mathrm{AN}$, and at least half of the remainder of the deaths have resulted from suicide. The peak occurrence of AN is during adolescence and early adulthood. Longitudinal studies have identified body dissatisfaction, dieting and picky eating as possible predictors for eating disorders. Additionally, multivariate studies have found obsessive-compulsive personality disorder as well as generalized anxiety indicate to have a strong predictive value for AN [3].
The exact psychogenesis of AN is unknown. Twin studies have indicated that monozygotic twins have significantly higher concordance rates than dizygotic twins for AN, indicating there is a genetic component to the development of AN [1]. Neurologic and hormonal changes associated with malnourishment may also play a role in the maintenance AN [6,7]. Additionally, deficits in dopamine and serotonin have been noted in AN patients: alterations in these neurotransmitters may play a role in eating behavior, mood and obsessional behavior [6]. An accurate way to conceptualize this is that, genetics loads the gun and environmental factors pull the trigger.

\section{Therapeutic role of RDs in refeeding}

Registered Dietitians (RDs) play an important role in the treatment of anorexia nervosa. RDs are responsible for initiating and advancing meal plans for weight restoration, monitoring weight trends and providing nutrition education to promote long-term successful outcomes. Along with the basic nutritional interventions for refeeding, along with the medical oversight, treatment plans also need to address the cognitive aspects of refeeding in patients with anorexia nervosa (AN) given the inherent mental struggles of this illness. Thus, RDs must incorporate a psychological or therapeutic technique to encourage the patient's progress during the refeeding process. Patients beginning the refeeding process often require substantial emotional support and motivation for increasing oral intake to a caloric amount sufficient to support consistent weight restoration [8]. In addition, nutrition education can be delivered in a manner that encourages the healing of the patients' relationship with food. A recent Delphi study assessing the

Correspondence to: Koutsavlis A, Eating Recovery Center, Denver, CO 80218, USA, Tel: 303-825-8578; Email: Ann.Koutsavlis@EatingRecovery.com

Key words: anorexia nervosa, refeeding syndrome, nutrition therapy, superior mesenteric artery syndrome, registered dietitian

Received: January 17, 2017; Accepted: February 15, 2017; Published: February 20,2017 
best practices for nutrition counseling in AN suggests that RDs should help the patients process their false nutrition beliefs and weight/body distortions and incorporate "motivational interviewing" to encourage the connection of mind and body [9].

Moreover, RDs often need to address limited food acceptance at the beginning stages of the refeeding process, as patients often enter treatment with a myriad of preexisting food rules and limitations tied to their eating disorder which are impediments to weight restoration [10]. These food restrictions are typically created by the patient from false nutrition beliefs, but can also be related to an emotional or physical trauma history. $\mathrm{RDs}$ are able to educate the patient on correct nutrition information while encouraging flexibility to incorporate the eliminated foods back into the patients' diet. This often necessitates beginning with smaller food exposures and working toward a normalized and balanced diet. These food exposures can be done in conjunction with the therapist or other members of the treatment team.

Food restrictions are sometimes masked by certain diets that may be recognized as socially acceptable or trending within modern day culture, such as vegetarianism, veganism, gluten-free or low-fat diets [11]. These diets create an easy way for patients to eliminate whole food groups based on a very strict and inflexible basis, for example a patient eliminating all foods with cheese or milk, while claiming to follow a vegan diet. Guidelines exist to address these dietary limitations, at an inpatient/residential level of care, which promote an "all foods fit" model, encouraging variety and flexibility in food choices upon admission. A study by Schebendach et al. assessed the correlation between variety in food selections and treatment outcome, and demonstrated that patients who chose a more varied diet while in treatment, including added sugars, fats, caloric beverages and starchy carbohydrates, had a better treatment outcome one year post-hospitalization [12]. It was also suggested that the inclusion of highly palatable foods encourages increased food intake and therefore improved outcomes with weight restoration. RDs and treatment teams, however, must be empathetic regarding the emotional difficulty for the patient to include foods previously eliminated from their diet. Working toward increased variety and inclusion of highly palatable foods should be addressed at each meeting with the $\mathrm{RD}$, while setting measurable goals for inclusion of fear or challenge foods.

Patients sometimes will also present with reported food allergies or intolerances, most commonly gluten/wheat and lactose/dairy, which further present the challenge of a limited caloric diet. RDs should therefore request medical documentation of these allergies. However, if no documentation is presented and the patient is unwilling to incorporate the eliminated foods, RDs should request testing for food allergies. Once results are available, false reports of allergens can be addressed by the RD and start to be incorporated into the diet. Even with confirmed negative testing for food allergies, patients can still have difficulty with food acceptance and require ongoing counseling within the scope of practice of the RD. If a patient has mild intolerances or reports adverse reactions to certain foods such as gastrointestinal discomfort, bloating, diarrhea or constipation, these symptoms may actually be a result of the physical refeeding process and not the food itself [8]. These gastrointestinal symptoms can be addressed by the medical doctor with medications or supplements like Lactase, so that the $\mathrm{RD}$ is able to continue addressing food acceptance.

\section{Guidance on refeeding}

Several professional organizations provide recommendations outlining appropriate refeeding protocols for patients with AN. The
Academy of Nutrition and Dietetics (AND) recommends replacement therapy for deficient electrolytes and fluids which must be initiated prior to refeeding in order to avoid metabolic complications of refeeding. Electrolyte monitoring for potassium, phosphorus, calcium, and magnesium should be done every day for the initial 3 days at the beginning of the refeeding process with repletion of inadequate levels as needed [13]. To avoid refeeding problems it is recommended by AND to begin feeding very conservatively at the beginning of the refeeding process. Starting caloric intake between $5-10 \mathrm{kcal} / \mathrm{kg} /$ day and advancing to $30 \mathrm{kcal} / \mathrm{kg} /$ day by day 8 is recommended by AND as well as the National Institute for Health and Clinical Excellence (NICE) $[13,14]$. In contrast, The American Psychiatric Association (APA) recommends 1-1.4 kg of weight gain per week for hospitalized patients. Meal plans should be initiated at $30-40 \mathrm{kcal} / \mathrm{kg} /$ day $(1,000-1600 \mathrm{kcal} /$ day) with advancements to $70-100 \mathrm{kcal} /$ day to promote adequate weight gain $[14,15]$. Clinical practice guidelines from Australia and New Zealand also support more aggressive approaches to refeeding starting at $1400-1500 \mathrm{kcal} /$ day with increases of $400-500 \mathrm{kcal} /$ day every 2-3 days [16].

Although many professional organizations have in the past recommended conservative approaches to refeeding, other studies support a more aggressive refeeding protocol. Current research concludes that previously used low caloric refeeding is too conservative and inefficient in mild and moderately malnourished patients with AN. In addition, higher caloric refeeding, when administered under medical monitoring, was not associated with increased risk of refeeding syndrome when electrolyte abnormalities were corrected [17]. Patients with higher caloric prescriptions, at the initiation of refeeding, experienced significantly faster weight gain and shorter hospital stays [18]. Another study determined that when a more conservative, low calorie refeeding approach was utilized, patients lost weight during the first week of refeeding due to inability for initial low caloric meal plans to meet the patients' basic energy needs [19]. When refeeding is approached too conservatively the "underfeeding syndrome", is the new term which is being popularized [20].

Several studies have also examined patient outcomes when more aggressive approaches to refeeding have been used. In a study by Neville et al. two groups of patients on low and high caloric refeeding protocols were compared. The higher caloric group had increases in BMI after day 1, but the lower caloric group did not until after day 2 . Although the higher caloric group initiated weight gain more quickly, the overall change in BMI between groups did not differ significantly. Rates of hypophosphatemia, hypomagnesemia, and hypokalemia also did not differ significantly between study groups, and the overall incidence of hypophosphatemia was associated with lower \%BMI on admission, but was not associated with caloric intake [21]. Garber et al. also compared the aggressive approach to a more conservative approach to refeeding. The higher caloric group's meal plans were advanced more than the lower caloric group $(122 \pm 8 \mathrm{kcal} /$ day vs $98 \pm 6$ $\mathrm{kcal} /$ day) resulting in more calorically adequate meal plans upon discharge. There were no cases of refeeding syndrome, and rate of weight gain in the higher caloric group was almost double that of the lower caloric group [22]. In another study, adolescent patients were started on 1,900-2,200 kcal/day, increasing their kcal to a minimum of $2,700 \mathrm{kcal} /$ day, with further increases as needed for continued weight restoration. Weight gain achieved in this study was $2.6 \mathrm{~kg}$ during the first two weeks of refeeding [23]. In addition, Madden et al's study also supports more aggressive refeeding using continuous nasogastric feeds for the first 24-72 hours of hospitalization. After the initial few days of refeeding, patients continued on nocturnal nasogastric feeds along 
with oral meal plans. This refeeding strategy resulted in robust weight gain in all patients in the first week of treatment, with an average weight gain of $5.1 \mathrm{~kg}$ after 2.5 weeks in treatment [24]. It is worth noting that in many of the aforementioned studies investigating the efficacy of more aggressive refeeding protocols, adolescent patients were being studied. Emerging research also resulted in positive outcomes when utilizing more aggressive refeeding protocols in adult patients. Redgrave et al. studied adult patients with AN who were started on 1,200-1,500 kcal/ day and rapidly advanced to $3,500-4,000 \mathrm{kcal} /$ day. No cases of severe hypophosphatemia were found, and patients successfully gained an average of $1.98 \mathrm{~kg} /$ week [25].

In addition to the initial caloric intake, it is important to also consider the macronutrient composition of meal plans during the refeeding process for patients with AN. However, current accepted practice is not based on rigorous research studies. In general, carbohydrates should be limited to $40-50 \%$ of total caloric intake in order to avoid exuberant increases in insulin secretion, leading to hypophosphatemia as well as postprandial hypoglycemia in a population with a proclivity for both complications $[26,27]$. In addition, excessive calories from dextrose may cause hepatic steatosis, or accumulation of fat in the liver [26]. Protein intake is typically around $1.5 \mathrm{grams} / \mathrm{kg}$ of body weight, and should only cautiously exceed $2 \mathrm{~g} / \mathrm{kg}$ [26]. Inclusion of essential fatty acids during the refeeding process is imperative, as neurological functioning, cell membrane functioning, and gene regulation rely on adequate lipid intake [28,29]. Macronutrients in the diet should come from a wide variety of calorically dense foods in order to meet patients' nutritional needs and promote variety in the diet. In order to make meal plans manageable for patients to consume and to avoid complaints of early satiety, total calories can be divided between three meals and three snacks per day, allowing for more normalized intake during the weight restoration process.

Although further research is needed, including research focusing on adult patients with $\mathrm{AN}$, many of the recent published studies support more aggressive approaches to refeeding than those previously suggested by the respected professional organizations. This field is clearly moving in the direction of higher initial caloric diets as well as faster increases in the subsequent caloric prescriptions. However, one population, who has yet to be formally studied, is patients with the most extreme degrees of AN, who have BMIs $<13 \mathrm{~kg} / \mathrm{m}^{2}$.

\section{Refeeding syndrome}

The refeeding syndrome is a potentially dangerous, but preventable, medical condition that can arise when reintroducing nutrition in a starved or malnourished patient. Refeeding syndrome can occur in a wide variety of populations including alcoholics, postoperative, homeless, and cancer patients, as well as those with AN upon resumption of caloric intake [30]. While there is no clear definition of refeeding syndrome, it can be described as a set of potentially serious medical complications, involving multiple body systems, with alternations in electrolytes, in malnourished patients who are now being fed orally, enterally, or parenterally [31].

Although refeeding syndrome is multi-faceted, hypophosphatemia is recognized as playing a central role in the onset of the syndrome. In general, total body phosphorus stores are depleted in starved patients, which are further exacerbated during the initial phase of refeeding. When the body begins to process carbohydrates, insulin is released, which causes glucose and phosphorus to move into the body's cells, thereby worsening the already present state of total body phosphorus deficiency [31]. Production of adenosine-5-triphosphate (ATP) and 2, 3-diphosphoglycerate (2,3 DPG), two important molecules necessary for energy production, is reduced as result thereof, leading to organ dysfunction, muscle cell death (rhabdomyolysis), acute respiratory and cardiac failure, tissue hypoxia, and hemolysis. In addition to hypophosphatemia, other electrolyte abnormalities may occur with the refeeding syndrome. These include decreases in serum potassium, magnesium, sodium, and thiamine levels, which can adversely impact the clinical stability of patients undergoing refeeding.

NICE has outlined guidelines, which establish two sets of criteria for identifying patients at high risk for refeeding syndrome (Table 1) [26,32]. Patients with one or more of the following are said to be at high risk for refeeding syndrome: $\mathrm{BMI}<16 \mathrm{~kg} / \mathrm{m}^{2}$, unintentional weight loss of $>15 \%$ in the past 3-6 months, little or no nutritional intake for $>10$ days, or low levels of potassium, phosphorus, or magnesium before refeeding. A patient with two or more of the following is also at high risk for refeeding syndrome: $\mathrm{BMI}<18.5 \mathrm{~kg} / \mathrm{m}^{2}$, unintentional weight loss of $>10 \%$ in the past 3-6 months, little or no nutritional intake from $>5$ days, and history of alcohol or drug misuse [32].

Recent data suggest that refeeding hypophosphatemia is directly correlated with the degree of weight loss, which has occurred as a result of the AN [26]. In addition, four initial reliable markers in determining those patients who may be at risk for refeeding hypophosphatemia include lower BMI, lower potassium, elevated hemoglobin, and lower prealbumin [30]. It is important to note that total caloric intake per se is not correlated with refeeding hypophosphatemia and the development of refeeding syndrome. Other risk factors for the development of refeeding hypophosphatemia have also been identified and include lower BMI, older age, and higher BUN upon admission [33]. Thus, it appears that the patients with $\mathrm{AN}$, who present with extreme low body weight, are at highest risk for the development of refeeding hypophosphatemia.

Three strategic treatment interventions play a role in preventing the refeeding syndrome including early identification of at risk patients, close laboratory monitoring, and administering an appropriate refeeding regimen [34]. It is recommended that patients be screened for risk of refeeding syndrome by obtaining a detailed history, clinical examination, and laboratory testing before the initiation of nutritional rehabilitation. The refeeding meal plan should be prescribed and managed by a $\mathrm{RD}$ and, as previously mentioned, the correct amount of calories to be initiated in $\mathrm{AN}$ is certainly gathering more recent interest. Electrolytes, phosphorus, and liver tests should be closely monitored every 1-2 days during the first 1-2 weeks of refeeding. If the patient develops abnormalities in phosphorus or other electrolytes, they must be aggressively repleted with oral supplements and monitored for additional time. On rare occasions, intravenous supplementation may be required when potassium is $<2.5$ $\mathrm{meq} / \mathrm{L}$ or phosphorus is $<2.1 \mathrm{meq} / \mathrm{L}[35]$.

Table 1. NICE guidelines for identification of patients at high risk for refeeding syndrome [26,32]

Patient has one or more of the following:

- BMI less than $16 \mathrm{~kg} / \mathrm{m}^{2}$

- unintentional weight loss greater than $15 \%$ within the last 3-6 months

- little or no nutritional intake for more than 10 days

- low levels of potassium, phosphate or magnesium prior to feeding.

Or patient has two or more of the following:

- BMI less than $18.5 \mathrm{~kg} / \mathrm{m} 2$

- unintentional weight loss greater than $10 \%$ within the last 3-6 months

- little or no nutritional intake for more than 5 days

- a history of alcohol abuse or drugs including insulin, chemotherapy, antacids or diuretics. 


\section{Managing gastrointestinal medical comorbidities}

There are a variety of GI medical comorbidities that may arise during the weight restoration process of patients with AN, which if untreated, can impact successful weight restoration. These include gastroparesis, superior mesenteric artery (SMA) syndrome, constipation, and celiac disease.

Gastroparesis, or delayed gastric emptying, presents with symptoms of early satiety, fullness, and nausea. This can be a significant barrier to treatment. Specifically, in AN, gastroparesis is related to diminished gastric capacity. Therefore, it is important that diet modifications be made to improve patient symptoms and ensure that adequate caloric needs are being ingested for weight restoration. In the initial stages of refeeding, more frequent and smaller volume meals improve gastroparesis symptoms [36]. However, these small, frequent meals can make it difficult to meet patient caloric needs. In this case, the utilization of liquid supplemental calories should be considered as liquids are less impacted by the reduced gastric size and improve symptoms [36]. Low fat and low fiber meals will also reduce symptoms of gastroparesis as both affect gastric emptying [36]. Temporary use of prokinetic agents such as metoclopramide and azithromycin may also be of utility.

SMA syndrome is another GI complication that can interfere with refeeding. It occurs as a result of the loss of the mesenteric fat pad normally situated between the SMA and the aorta leading to the compression of the third portion of the duodenum [37]. The loss of the fat pad is directly due to significant weight loss. While the prevalence of SMA syndrome is rare $(0.013-3.0 \%$ in the general population), this complication has been observed in AN patients with severe malnutrition [37]. Symptoms include nausea, vomiting, postprandial abdominal pain early in the course of eating, and early satiety. The diagnosis of SMA syndrome may be delayed as many of the symptoms or patient complaints are commonly associated with weight restoration as well as being inherent to the psychiatric part of AN. SMA syndrome is diagnosed by an upper GI study, or by an abdominal CT scan. The primary treatment for SMA syndrome is aimed at weight restoration in order to restore the mesenteric fat pad [37]. This can be achieved by nutritional therapy including a liquid or soft oral diet of small particle sized foods, enteral nutrition via nasogastric tube, or percutaneous endoscopic gastrostomy/jejunostomy (PEG/PEJ) tubes.

Constipation is also a common complaint of most patients with AN. This is typically due to a restriction of caloric intake, weight loss, and slowed colonic motility due to reflex hypofunctioning of the bowel [38]. With weight restoration, colonic motility improves and constipation resolves [38]. Ensuring adequate fluid intake can also improve symptoms of constipation. However, when needed, the preferred treatment of constipation is the use of osmotic laxatives [38].

Celiac disease is an autoimmune disease in which the dietary intake of gluten, found in wheat, barley, and rye, triggers an inflammatory response in genetically predisposed individuals [39]. Around $1 \%$ of the general population has celiac disease, with a 2 times higher prevalence in women. In recent years, there has been an increased interest in a potential link between celiac disease and eating disorders. However, research fails to show an increased prevalence of celiac disease among AN patients. One recent study indicates that individuals with celiac disease have similar eating behavior characteristics as those with eating disorders [39]. There is insufficient evidence to support routine screening for celiac disease in AN patients. Patients with both AN and/or celiac disease may complain of similar symptoms- diarrhea, constipation, abdominal pain, bloating, and vomiting. This emphasizes the need to use caution when making dietary accommodations without a confirmed diagnosis of celiac disease in patients with AN. If abdominal complaints remain after treatment of AN, the patient may then benefit from celiac disease testing.

\section{Summary}

In summary, refeeding the patient with $\mathrm{AN}$ is a critical part of the overall treatment plan. However, refeeding can be fraught with many medical complications. Most of these are preventable if judiciously considered early on in the refeeding process. The RD is an integral member of the multidisciplinary team which should be involved with the AN patient in order to effectuate a successful process of refeeding and natural rehabilitation. Weight restoration is a key predictor of sustained recovery in AN.

\section{References}

1. Mosquera D, Gonzalez A, Leeds AM (2014) Early experience, structural dissociation, and emotional dysregulation in borderline personality disorder: the role of insecure and disorganized attachment. Borderline Personal Disord Emot Dysregul 1: 15. [crossref]

2. Lock JD, La Via M, Rhe American Academy of Child and Adolescent Psychiatry (AACAP) Committee on Quality Issues (2015) Practice parameter for the assessment and treatment of children and adolescents with eating disorders. $J$ Am Acad Child Adolesc Psychiatry 54: 412-425. [crossref]

3. Rosenvinge JH, Pettersen G (2015) Epidemiology of eating disorders part II: an update with a special reference to the DSM-5. Adv Eat Disord 3: 198-220.

4. Norris ML, Apsimon M, Harrison ME, Obeid N, Buchholz A, et al. (2012) An examination of medical and psychological morbidity in adolescent males with eating disorders. Eat Disord 20: 405-415. [crossref]

5. Sullivan PF (1995) Mortality in anorexia nervosa. Am J Psychiatry 152: 1073-1074. [crossref]

6. Phillipou A, Rossell SL, Castle DJ (2014) The neurobiology of anorexia nervosa: a systematic review. Aust N Z J Psychiatry 48: 128-152. [crossref]

7. Muller MJ, Enderle J, Pourhassan M, Braun W, Eggeling B, et al. (2015) Metabolic adaptation to caloric restriction and subsequent refeeding: the Minnesota Starvation Experiment revisited. Am J Clin Nutr 102: 807-819. [crossref]

8. Arthur B, Strauss L, Mehler PS (2015) Refeeding the patient with anorexia nervosa: perspectives of the dietitian, psychotherapist and medical physician. World Journal of Nutrition and Health 3: 29-34

9. Mittnacht AM, Bulik CM (2015) Best nutrition counseling practices for the treatment of anorexia nervosa: a Delphi study. Int J Eat Disord 48: 111-122. [crossref]

10. Cockfield A, Philpot U (2009) Feeding size 0: the challenges of anorexia nervosa Managing anorexia from a dietitian's perspective. Proc Nutr Soc 68: 281-288. [crossref]

11. Bardone-Cone AM, Fitzsimmons-Craft EE, Harney MB, Maldonado CR, Lawson MA, et al. (2012) The inter-relationships between vegetarianism and eating disorders among females. J Acad Nutr Diet 112: 1247-1252. [crossref]

12. Schebendach JE, Mayer LE, Devlin MJ, Attia E, Contento IR, et al. (2011) Food choice and diet variety in weight-restored patients with anorexia nervosa. J Am Diet Assoc 111 : 732-736.

13. Ozier AD, Henry BW (2011) Position of the American Dietetic Association: nutrition intervention in the treatment of eating disorders. J Am Diet Assoc 111: 1236-1241. [crossref]

14. National Institute for Health and Clinical Excellence (2006) Nutrition support in adults: Oral nutrition support, enteral tube feeding and parenteral nutrition. Clinical guideline [CG32].

15. Yager J, Devlin MJ, Halmi KA, Herzog DB, Mitchell JE, et al. (2006) Practice guidelines for the treatment of patients with eating disorders. 3rd ed. Washington, DC: American Psychiatric Association.

16. Hay P, Chinn D, Forbes D, Madden S, Newton R, et al. (2014) Royal Australian and New Zealand College of Psychiatrists clinical practice guidelines for the treatment of eating disorders. Aust NZ J Psychiatry 48: 977-1008. [crossref]

17. Garber AK, Sawyer SM, Golden NH, Guarda AS, Katzman DK, et al. (2016) A systematic review of approaches to refeeding in patients with anorexia nervosa. Int $J$ Eat Disord 49: 293-310. 
18. Garber AK, Michihata N, Hetnal K, Shafer MA, Moscicki AB (2012) A prospective examination of weight gain in hospitalized adolescents with anorexia nervosa on a recommended refeeding protocol. J Adolesc Health 50: 24-29. [crossref]

19. Katzman DK (2012) Refeeding hospitalized adolescents with anorexia nervosa: is "start low, advance slow" urban legend or evidence based? J Adolesc Health 50: 1-2.

20. Marikar D, Reynolds S, Moghraby OS (2016) Junior MARSIPAN (Management of Really Sick Patients with Anorexia Nervosa). Arch Dis Child Educ Pract Ed 101: 140143. [crossref]

21. Golden NH, Keane-Miller C, Sainani KL, Kapphahn CJ (2013) Higher caloric intake in hospitalized adolescents with anorexia nervosa is associated with reduced length of stay and no increased rate of refeeding syndrome. J Adolesc Health 53: 573-578.

22. Garber AK, Mauldin K, Michihata N, Buckelew SM, Shafer MA, et al. (2013) Higher calorie diets increase rate of weight gain and shorten hospital stay in hospitalized adolescents with anorexia nervosa. $J$ Adolesc Health 53: 579-84. [crossref]

23. Whitelaw M, Gilbertson H, Lam P-Y, Sawyer SM (2010) Does aggressive refeeding in hospitalized adolescents with anorexia nervosa result in increased hypophosphatemia? J Adolesc Health 46: 577-82. [crossref]

24. Madden S, Miskovic-Wheatley J, Clarke S, Touyz S, Hay P, et al. (2015) Outcomes of a rapid refeeding protocol in Adolescent Anorexia Nervosa. J Eat Disord 3: 8. [crossref]

25. Redgrave GW, Coughlin JW, Schreyer CC, Martin LM, Leonpacher AK, et al. (2015) Refeeding and weight restoration outcomes in anorexia nervosa: challenging current guidelines. Int J Eat Disord 48: 866-73.

26. Mehler PS, Winkelman AB, Andersen DM, Gaudiani JL (2010) Nutritional rehabilitation: practical guidelines for refeeding the anorectic patient. J Nutr Metab 2010: 625782 .

27. Kohn MR, Madden S, Clarke SD (2011) Refeeding in anorexia nervosa: increased safety and efficiency through understanding the pathophysiology of protein calorie malnutrition. Curr Opin Pediatr 23: 390-394.
28. Marzola E, Nasser JA, Hashim SA, Shih PA, Kaye WH (2013) Nutritional rehabilitation in anorexia nervosa: review of the literature and implications for treatment. $B M C$ Psychiatry 13: 290. [crossref]

29. Reiter CS, Graves L (2010) Nutrition therapy for eating disorders. Nutr Clin Pract 25: 122-136. [crossref]

30. Brown CA, Sabel AL, Gaudiani JL, Mehler PS (2015) Predictors of hypophosphatemia during refeeding of patients with severe anorexia nervosa. Int J Eat Disord 48: 898-904.

31. Mehler PS, Crews CK (2001) Refeeding the patient with anorexia nervosa. Eat Disord 9: 167-171. [crossref]

32. National Institute for Health and Clinical Excellence (2009) Guideline for the Management of Refeeding Syndrome (Adults). 2nd ed. NHS Foundation Trust.

33. Kameoka N, Iga JI, Tamaru M, Tominaga T, Kubo H, et al. (2016) Risk factors for refeeding hypophosphatemia in Japanese inpatients with anorexia nervosa. Int $J$ Eat Disord 49: 402-06.

34. Tresley J, Sheean PM (2008) Refeeding syndrome: recognition is the key to prevention and management. J Am Diet Assoc 108: 2105-2108. [crossref]

35. Arthur B, Strauss L, Mehler PS (2015) Refeeding the patient with anorexia nervosa: perspectives of the dietitian, psychotherapist and medical physician. World Journal of Nutrition and Health 3: 29-34.

36. Mascolo M, Geer B, Feuerstein J, Mehler PS (2016) Gastrointestinal comorbidities which complicate the treatment of anorexia nervosa. Eat Disord 21: 1-12. [crossref]

37. Mascolo M, Dee E, Townsend R, Brinton JT, Mehler PS (2015) Severe gastric dilatation due to superior mesenteric artery syndrome in anorexia nervosa. Int $J$ Eat Disord 48: 532-534

38. Mehler PS, Krantz MJ, Sachs KV (2015) Treatments of medical complications of anorexia nervosa and bulimia nervosa. J Eat Disord 3: 15. [crossref]

39. Passananti V, Siniscalchi M, Zingone F, Bucci C, Tortora R, et al. (2013) Prevalence of eating disorders in adults with celiac disease. Gastroenterol Res Pract: 491657. [crossref]

Copyright: $\odot 2017$ Koutsavlis A. This is an open-access article distributed under the terms of the Creative Commons Attribution License, which permits unrestricted use, distribution, and reproduction in any medium, provided the original author and source are credited. 